Poetry

\title{
Nutritional chiefdom
}

\section{Poetry}

To go up of the wisdom

We must remove the boredom

It is all about nutrition

That works for all the chiefdom

Dinner lunch and breakfast

Are what we used to eat fast

They but are a multiple

Source of crucial cast

Modest frequent meals

Should be today's drug pills

Avoid large meals overnight

To pay only less of bills

Do exercise physically

No monthly or the weekly

Run it daily for the time

You eat none of mimickly

Comes in the end practice

All of the rest the entice

You'd deserve a success

Should be good at prentice. ${ }^{1-25}$

\section{Acknowledgements}

None.

\section{Conflict of interest}

Author declares that there is no conflict of interest.

\section{References}

1. Nikkhah A. Improving Human Health through Optimizing Food Intake and Exercise Time Management: A Real-World Science. J Nutr Health Food Eng. 2015;2(5):00070.

2. Nikkhah A. Exercise into Size. J Nutr Health Food Eng. 2015;2(5):00071.

3. Nikkhah A. Nutritional Health: Pool \& Plunger. J Nutr Health Food Eng. 2015;2(5):00069.

4. Nikkhah A. Nutrition is Ambition. $J$ Nutr Health Food Eng. 2015;2(5):00068.

5. Nikkhah A. Diabesity and Lifestyle. Curr Res Diabetes Obes J. 2015;1(1):CRDOJ.MS.ID.555552.
Special Issue - 2015

\section{Akbar Nikkhah}

Department of Animal Sciences, University of Zanjan, Iran

Correspondence: Akbar Nikkhah, Chief Highly Distinguished Professor, Department of Animal Sciences, Faculty of Agricultural Sciences, University of Zanjan, Foremost Principa Highly Distinguished Elite-Generating Scientist, National Elite Foundation, Iran, Email anikkha@yahoo.com

Received: October 7, 2015 | Published: October 7, 2015

6. Nikkhah A. Gestational Fetal Genomics Bioengineering to Improve Individual and Public Health. Aus J Biotechnol Bioeng. 2015;2(4):1050.

7. Nikkhah A. A Time-Efficient Exercise Formula for Normalizing Obese Body Mass Index. Adv Obes Weight Manag Control. 2015;3(2):00049.

8. Nikkhah A. Lifestyle Optimization: Today's Foremost Probiotic. J Probiotics Health. 2015;3(2):e119.

9. Nikkhah A.Vital Frontiers of Science Education: Global Obligations. Adv Crop Sci Tech. 2015;3:e126.

10. Nikkhah A. Improving Life Quality via Circadian Timing of Nutrient Intake: Linking Ruminant Agriculture to Human Health. EC Agriculture. 2015;2(1):258-259.

11. Nikkhah A. Optimized Lifestyle via Timing of Food Intake: Bridging Ruminant Agriculture to Human Health. J Food Nutri. 2015;2(2):013.

12. Nikkhah A. Scheduling Exercise-Eating to Optimize Gut Probiotics: A Global Invention. J Probiotic Health. 2015;3:e118.

13. Nikkhah A. A Realistic Weight Management Nutritional Regimen for Today's Life. J Nutr Health Food Eng. In Press. 2015.

14. Nikkhah A. Leading Edges of Economy-Building Science Education. $J$ Glob Econ. 2015;3:e109.

15. Nikkhah A. Circadian Optimization of Fruit and Vegetable Intake: A Gut-Exerciser Probiotic. J Prob Health. 2015;3(2):e116.

16. Nikkhah A. Safety against Diabetes via Optimizing Circadian Intake Patterns: Science Evolution Cropped. Adv Crop Sci Technol. 2015;S1:e001.

17. Nikkhah A. Establishing Regular Patterns of Cellular Mechanics to Minimize Oncogenesis: Animal Sciences Inspire. Aust J Vet Sci Anim Husb. 2015;2(2):1010.

18. Nikkhah A. The Art of Manipulating Nutrient Bioprocessing In Ruminants: Behind the Rumen Wheel. $J$ Bioprocess Biotech. 2015;5:e134.

19. Nikkhah A. Towards a Global Anti-Diabetes Exercise Program. J Bioprocess Biotechniq. 2015;5:e135.

20. Nikkhah A. Standardizing Appetite through Timing of Food Intake to Minimize Metabolic Disorders: A Veterinary Revelation. J Veterinar Sci Technol. 2015;6:e116.

21. Nikkhah A. Slowing Aging via Joint Rhythmic Exercise and Optimized Eating Behavior: Nature Enthuses. J Bioprocess Biotech. 2015;5:e136. 
22. Nikkhah A. Lifestyle Bioengineering via Scheduled Intake: Bridging Animal Agriculture to Human Medicine. Aust J Biotechnol Bioeng. 2015;2(3):1045.

23. Nikkhah A. Pragmatic Science Edification: The Evolving Biodiverse Brain of Society. J Biodivers Biopros Dev. 2015;2:e109.
24. Nikkhah A. Matching Substrate Provision and use to Power Cut Oncogenesis. J Cancer Prev Curr Res. 2015;3(2):71.

25. Nikkhah A. Circadian Timing and Regularity of Physical Activity: A Novel Bioprocess to Prevent Devastating Modern Diseases. J Bioprocess Biotechniq. 2015;5:e131. 